\title{
18th IUPAC International Symposium on Macromolecular Metal Complexes (MMC-18)
}

The 18th IUPAC International Symposium Macromolecular Metal Complexes (MMC-18) will be held on June 10-13, 2019, on a comfortable passenger ferry "Nekrasov", plying the route Moscow-Tver-Myshkin-Uglich-Moscow.

\section{Organizers}

Lomonosov Moscow State University

Russian Academy of Science (RAS)

Chairman Professor E. Karakhanov, Lomonosov Moscow State University, Russia

Co-chairman Professor A. Maximov, A. V. Topchiev Institute of Petrochemical Synthesis, RAS, Russia

\section{Organizing Committee}
A. V. Anisimov
A. V. Hakobyan
Yu. S. Kardashev
V. A. Vinokurov
G. I. Dzhardimalieva
A. P. Glotov
L. A. Kulikov
A. V. Vutolkina
S. V. Yegazaryants
D. N. Gorbunov
M. V. Terenina

\section{Program Committee}

Chairman

Professor A. L. Maximov
A. V. Anisimov
Yu. S. Kardashev
M. V. Terenina
E. R. Badamshina
S. V. Lyulin
A. A. Yaroslavov

A. G. Dedov

International Advisory Board
P. A. G. Cormack (UK)
G. I. Dzhardimalieva (Russia)
R. B. Grubbs (USA)
A. Guiseppi-Elie (USA)
K. Hanabusa (Japan)
A. Haryono (Indonesia)
M. Higuchi (Japan)

E. A. Karakhanov (Russia)

N. Kimizuka (Japan)

A. L. Maximov (Russia)

S. Kudaibergenov

B. L. Rivas (Chile)

(Kazakhstan)

E. Rosenberg (USA)

K. Levon (USA)

Ch. K. Luscombe (USA)

H. Tenhu (Finland)

A. W. Trochimczuk (Poland)

H. Nishide (Japan)

K. Yamamoto (Japan)

\section{Scientific Program}

Plenary and sectional meetings with lectures and oral reports and poster sessions are scheduled in the frames of the event. They will cover fundamental aspects of Chemistry of Macromolecular Metal Complexes:

- Macromolecular Metal Complexes - Catalysis and Metal Capture;

- Organic-Inorganic Hybrids, Metallopolymers;

- Energy, Electronic, Optical, Photo- and Magnetic Applications;

- Supramolecular Chemistry, Self-Assembly and Polymer Complexes;

- Biomaterials and Biological Applications.

\section{Contacts:}

Scientific Program: Prof. Anton Maximov, e-mail: mmc-18@yandex.ru; tel: +7 (495) 9395377.

Sponsorship and Participation in the Exhibition: Darya Andreeva, tel. +7 (499) 390 3438, +7 (926) 891 7623;

e-mail: chphconf@yandex.ru.

Technical Support (any questions regarding online registration, hotel reservation, field excursions (tours) and social program, payment of registration fees, abstract submission, transfers etc.), Technical Secretariat of the Symposium, Ida Baykova, tel.: +7 (926) 848 2358; +7 (499) 390 3438; fax: +7 (499) 137 3479; e-mail: mmc-18@confreg.org.

\section{http://www.mmc-18.org}

